\title{
Integrated Applications with Laser Technology
}

\author{
Octavian DOSPINESCU ${ }^{1}$, Paul BRODNER ${ }^{2}$ \\ ${ }^{1}$ Faculty of Economics and Business Administration, AL.I.Cuza University, Iasi \\ ${ }^{2}$ OSF Global Services \\ doctav@uaic.ro,paulbrodner@gmail.com
}

\begin{abstract}
The introduction of new materials as Power Point presentations are the most convenient way of teaching a course or to display a scientific paper. In order to support this function, most schools, universities, institutions, are equipped with projectors and computers. For controlling the presentation of the materials, the persons that are in charge with the presentation use, in most cases, both the keyboard of the computer as well as the mouse for the slides, thing that burdens, in a way, the direct communication (face to face) with the audience. Of course, the invention of the wireless mouse allowed a sort of freedom in controlling from the distance the digital materials. Although there seems to appear a certain impediment: in order to be used, the mouse requires to be placed on a flat surface. This article aims at creating a new application prototype that will manipulate, only through the means of a light-beam instrument (laser fascicle), both the actions of the mouse as well as some of the elements offered by the keyboard on a certain application or presentation. The light fascicle will be ,connected" to a calculus system only through the images that were captured by a simple webcam.
\end{abstract}

Keywords: Surface Emitting Lasers, Technology Management, Software Prototyping, Optical Recognition Software

1 Introduction

We are now in the information and knowledge age and the ways data can be presented are very different [1]. The laser pointers are usually used to attract the attention on some important aspects within the presentation. They also were incorporated in wireless mice, but using such an instrument offers only a simple navigation control and it cannot be used for some more complex applications. The functional requirements aim at the simplification of the interaction between manmachine (computer), especially with the help of a webcam and with the help of a laser device. The application will be installed on any computer that has a webcam. Other than a webcam, the user has to have a laser device (laser pointer, or any other powerful light fascicle). Once launched, the application will „read" through the webcam the images that were captured from a certain location that was previously established (example: at a presentation where it is used a projector).

Through the means of some processing algorithms on the captured images, the action developed by the movement of the laser point on the board, will be transmitted to the com- puter, more exactly to the mouse. In other words, the application will use only one laser device that will allow the user to control the movement and the actions of the mouse from a far distance.

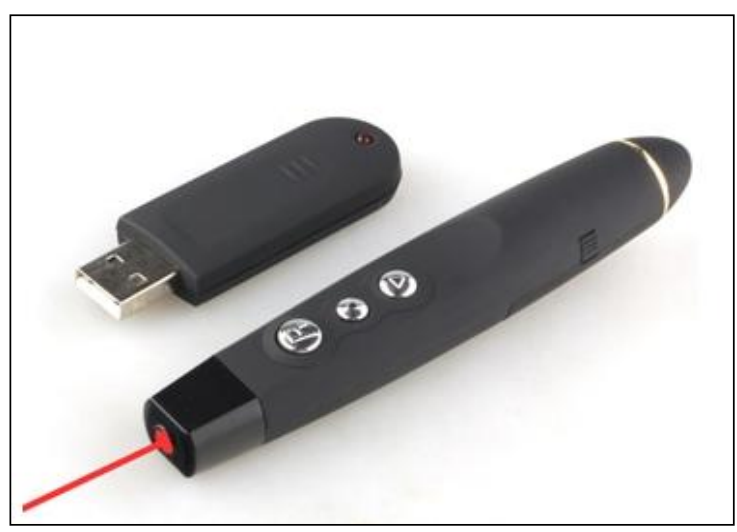

Fig. 1. Laser Pointer

The general operational way can be synthesized in the following important steps:

- The user is the one that will start the application and who will initialize the main way, meaning the verification of the existence of a webcam, the installation of the specific drivers of the webcam, reading the configuration files; 
- Starting the webcam will automatic initiate the capturing of the images (if this option was marked by the user). At this moment will be launched two wires of execution: after the processing of the image by the webcam, a web framed is saved as an image.

- The captured frame is submitted to optimization algorithms for making more effective the detection process of the laser point.

- The Laser Module will instantiate the detection process of the light fascicle, which through the means of some algorithms will give back and it will graphically display the location of the laser fascicle; moreover the mouse of the computer will be repositioned at a new coordinate.

- The Mouse Module is the one that will control the actions developed by the laser point, the actions of the mouse as well as the contextual graphic menu.

The application will interact with any other program that is installed on the computer. Some specific gestures could be memorized by the application, in order to use a certain order (for example: if the laser point will move to the right in the upper part of the board (N-E) Microsoft Office Outlook will open) as well as the various actions of the mouse: single/double left/right clock, saved settings in a configuration file.

\section{Hardware configuration and software development}

Regarding the hardware resources that are concerned, the prototype proposed is composed from the following:

- Laptop or PC having as operating systems Windows XP/VISTA/7 (the other operating systems were not tested);

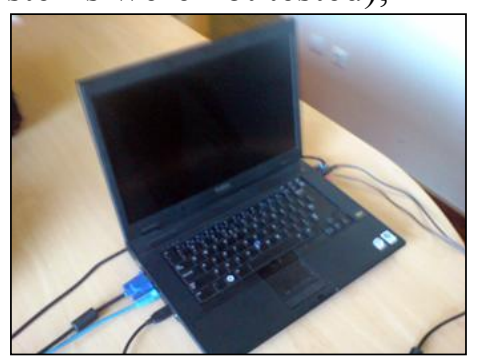

Fig. 2. Laptop/computer
- A projector that has to be connected to the computer (is an optional instrument; the application may work even if this device is missing);

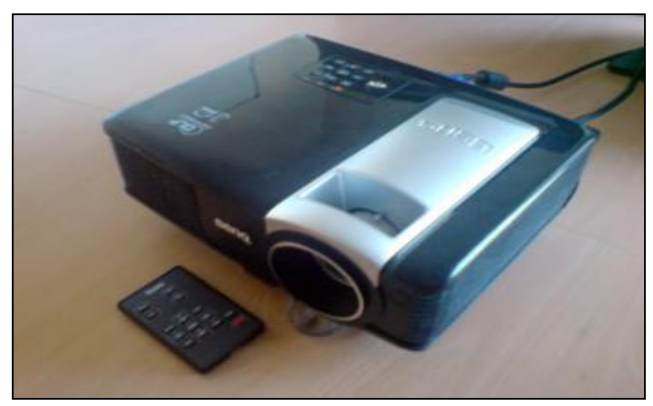

Fig. 3. Projector

- Integrated webcam or a webcam connected through a USB (the application offers the possibility to choose one of the available cameras);

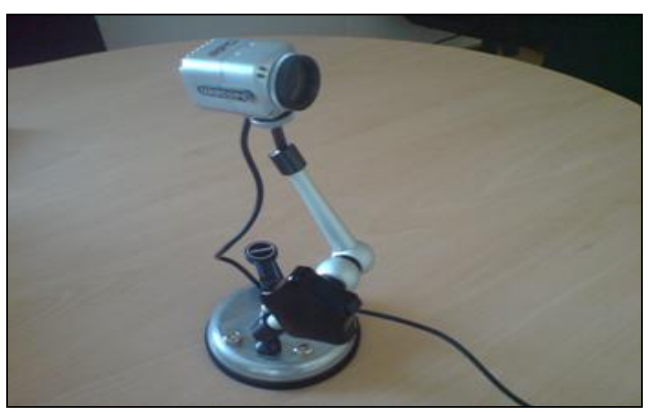

Fig. 4. Webcam

- A laser pointer

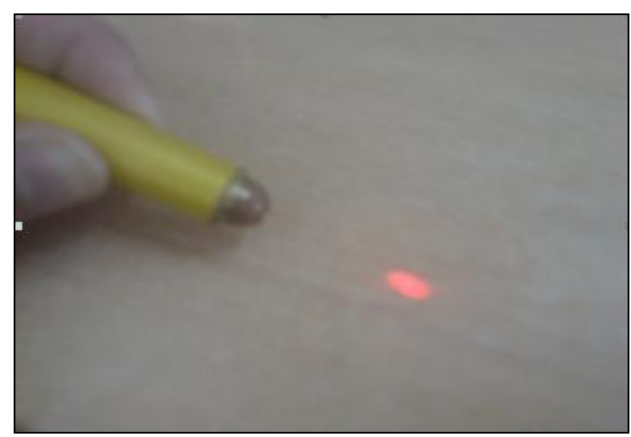

Fig. 5. Laser pointer

Only a part of the requirements from above are compulsory. Others like: graphic projector or laser pointer (another instrument may be used that generates a powerful light fascicle) may be missing. 


\section{Implemented System}

Through this paper we aim at presenting the determination algorithm of the existence of a laser fascicle from a given image. In our way we decided that the control instrument of the mouse to be a LASER (Light Amplification by Stimulated Emission of Radiation) because of the special characteristics that it holds [2], [3]:

- It is monochromatic: the wave length presents only one color. Even though some lasers may generate various wave lengths, the emitted light is very pure being composed from one color of the visual specter.

- It is directed: traverses a big distance on one trajectory.
- It is coherent: has the same wave length and a constant phase in time.

It is necessary to understand the operational process of a laser in order to develop valid algorithms. In what the mono-chromatisation of the light fascicle is concerned, the color is determined by the frequency or by the wave length. The smaller wave lengths are the ultraviolet ones (under 400 nanometers $-\mathrm{nm}$ ) but these are not visible for the human eye, in other words they are not in the visible electromagnetic specter. The human eye is able to distinguish length waves on a scale from 390 and $750 \mathrm{~nm}$, according to [4]. This specter does not have all the colors that the human eye can see. Figure 6 shows the colors that are visible for the human eye according to the wave length.

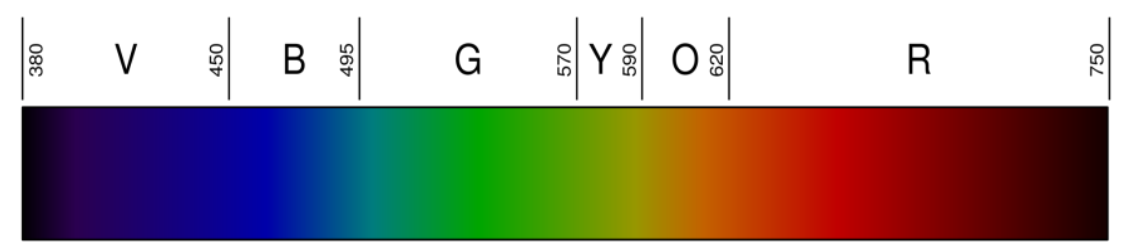

Fig. 6. Visible linear specter of color on wave lengths (nm)

Knowing this information, an efficient method is the detection of the laser fascicle that is based on one of its characteristics: emitted color (monochromatic). Being given a certain image, the light point (Red, Green) may be easily detected; Bowden [5] even explains in his paper some methods of object detection, movement detection, being used the captured colors.

If we take into account the fact that our application is developed especially for presentations, where there are used a various range of colors, this solution is not a very convenient one. In order to solve this problem it was taken into account the light offered by the laser point. In conditions of different shades of light, it was noticed that the point of the laser has always the highest brightness in an image.

An image represents a replica of an object and it is considered to be a function of two real variables $\mathrm{x}$ and $\mathrm{y}$ [6]. It is composed from many points or pixels [7] - the smallest component of a digital image that may be controlled or modified. Each pixel has an address that corresponds with a coordinate and represents a very small part from the whole picture (it is like in the case of puzzles). These pixels are characterized through position, intensity and time (in case of films). In all the combinations these parameters define the static images or the video clips. As long as there are used more pixels (high resolution), the final image will look more realistic. And therefore we get to the main characteristic in the case of solving our algorithm. The number of distinct colors may be represented by a pixel, depends on the number of bits on the pixels (bpp - bits per pixel):

- An image with 1 bpp will use only 1 bit for each pixel, therefore each pixel will beat a certain point 1 or 0 . Each bits adding will enlarge the number of available colors.

- An image with 2 bpp will have 4 colors (2 $\mathrm{x} 2)$, an image with 3 bpp $(2 \times 2 \times 2)$ will have 8 colors, and so on. 
Our algorithm will be applied to our images with 24 bpp, meaning 16.8 million colors. Therefore, for an efficient manipulation of the information that exists in an image, it was decided that the keeping of the pixels to be saved in the memory under a onedimensional matrix. All the information of an image - both on the $\mathrm{X}$ as well as on the $\mathrm{Y}$ axis - will contain all the pixels that form the length/width of the image. These pixels are saved like numbers from 0 to 255, meaning colors like Red, Green, Blue, as well as their opacity. Brightness $=(299 *$ Red $+587 *$ Green +
$114 *$ Blue $) / 1000$

According to W3C [8], the brightness can be calculated according to the RGB (Red/Green/Blue) of a pixel from an image, with the formula from above. Also, it is very important to detect the right position of every pixel [9]. Further on we will present a small example in order to describe the algorithm: the figure from below presents the information of all the pixels of a static image.

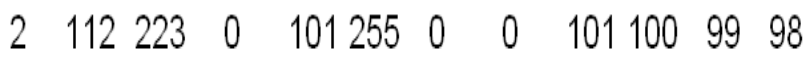 \\ $112223245 \quad 245001222222268 \quad 78 \quad 78 \quad 199$

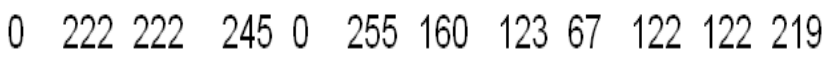

Fig. 7. The information of the pixels of an image saved in an array

As it can be seen, this array has 12 columns and 3 lines but the color of a pixel is represented by 3 cells (RGB) therefore the first 3 cells $(2,112$ and 223) are the colors of the first pixel, $(0,101$ and 255) of the second pixel, and so on. The problem consist in the fact that all the information is saved in a onedimensional array and its course will be done from the first position (2) up to the last (219) not taking into account the length or the width.
On the basis of the previous mentioned formula, we will calculate the most powerful brightness of the existing pixels, using also the ColorMatrix class [10], [11]. If we will find a $\mathrm{n}$ number of pixels with a brightness higher than 241 (an initial value that can be modified by the user; the most powerful brightness $=255$, the smallest $=0$ ) we will keep the current position of the pixel in the one-dimensional array. Example: if the pixel $(112,223$, and 245) is the brightness, its position will be kept (15).

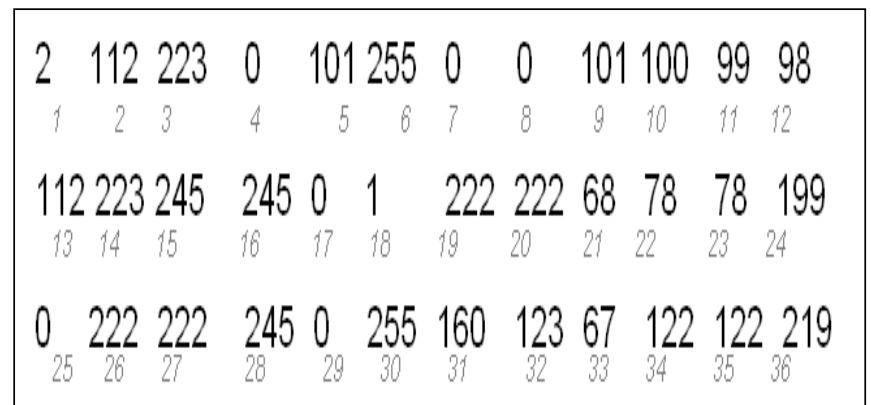

Fig. 8. Pixels and adjacent positions in the same order like they are saved in the onedimensional array

According to this information and taking into account the fact that the width of the 12 pixels image, the coordinates of the cell can be calculated with accuracy $(112,223$ and 245) $(x=1 ; y=2)$. 


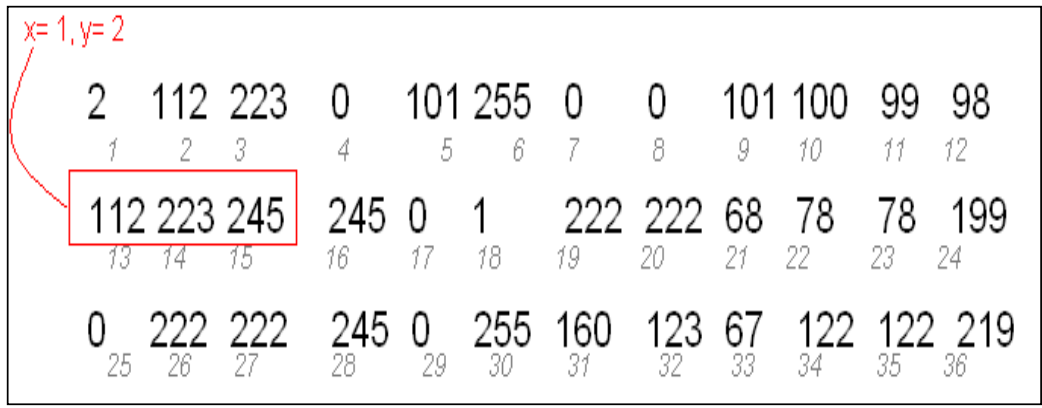

Fig. 9. Computing the coordinates

The practical implementation of the theoretical notions from above is presented in the listing from below, using a Visual Basic
.NET implementation based on several rules [12], [13], [14]:

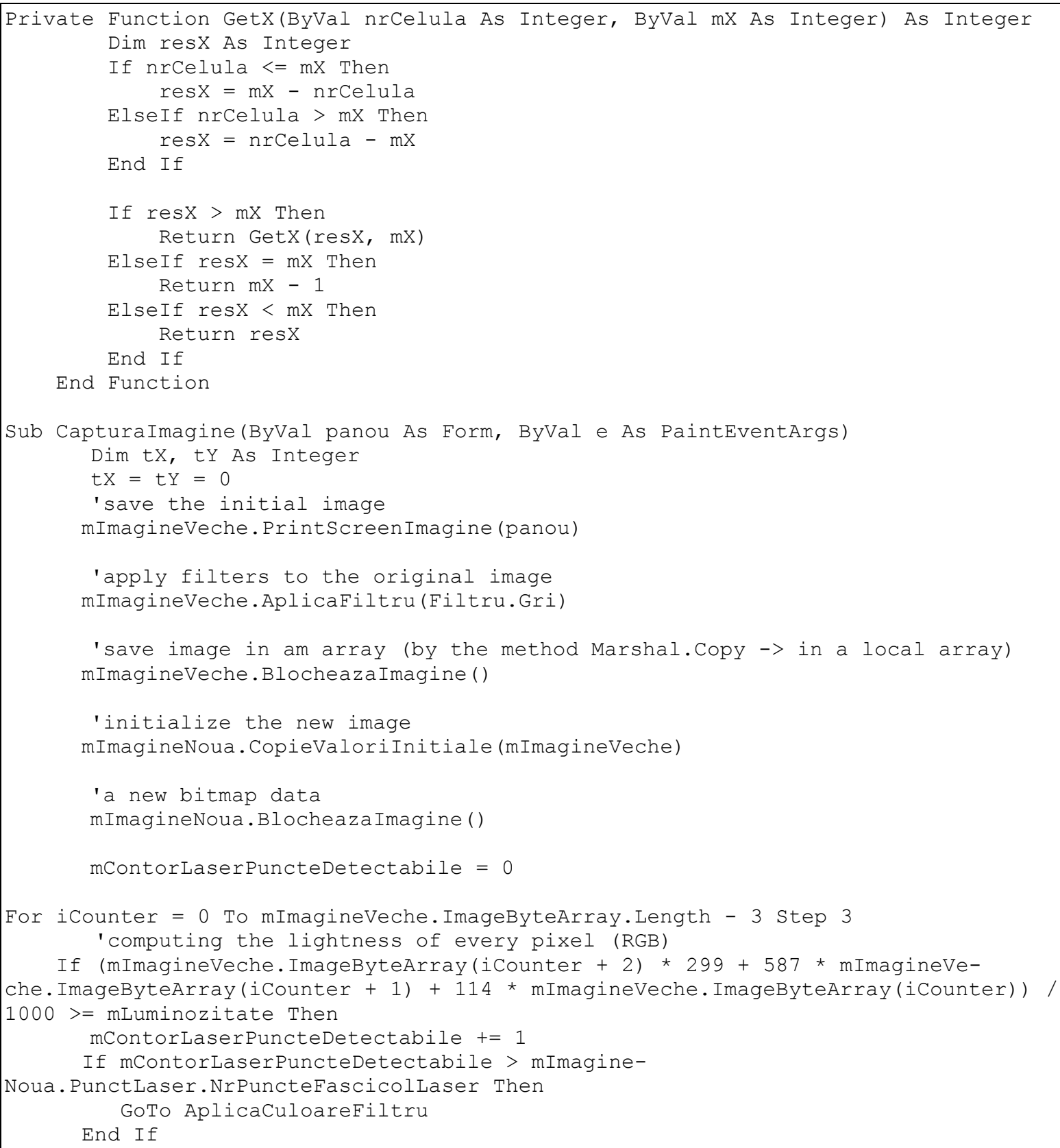




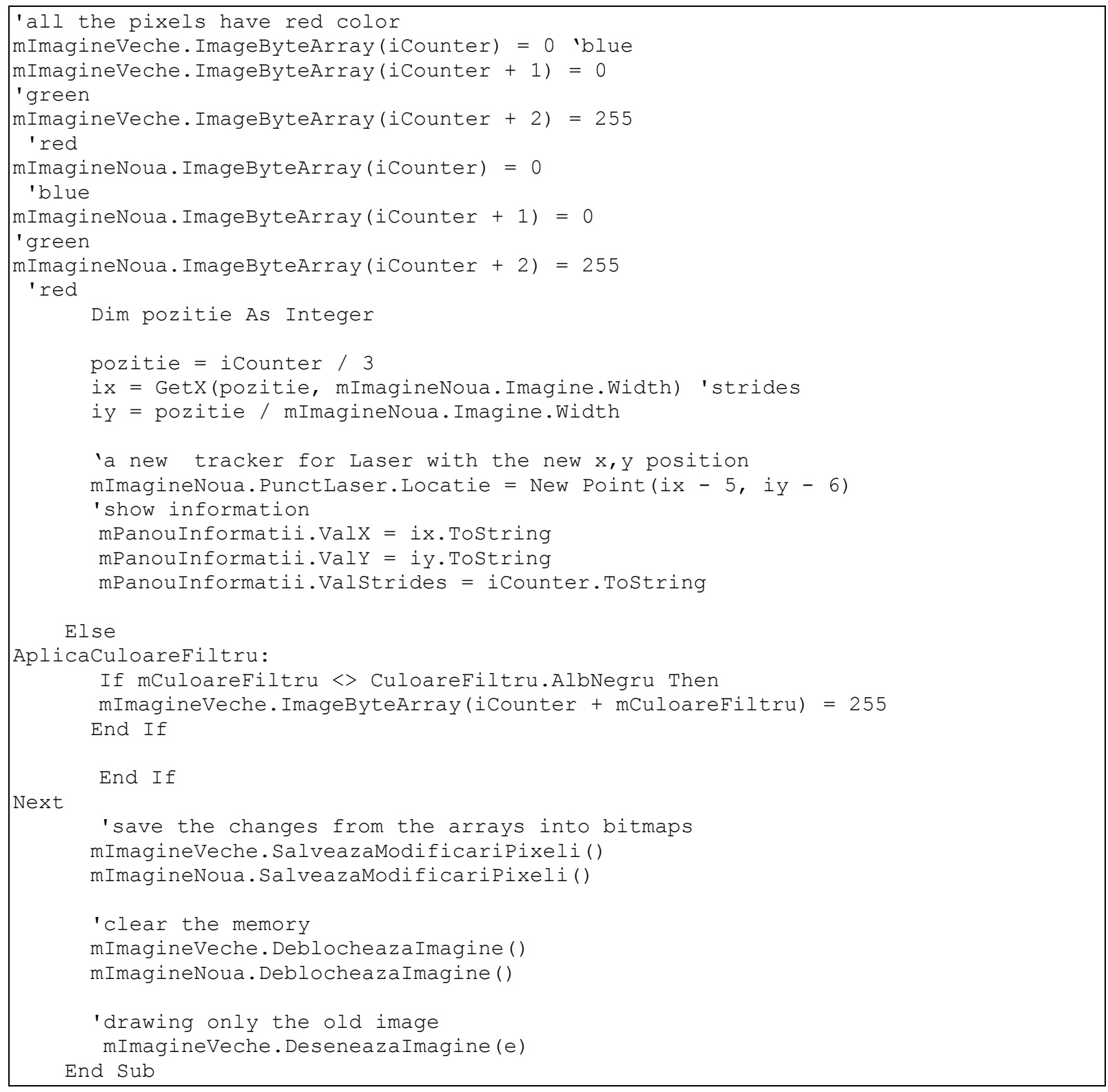

The above algorithm will calculate, according to brightness, the fascicle from an image. Of course, these calculations will be done in a very short time (according to the power of calculation of the computer) for each image/frame captured by the webcam. The new image will be displayed to the user only with the graphic representation of this point.

4 The Interface of the Application and Future Directions

The interface of the application has the following main functions:

1.1 - selecting an available webcam;

1.2 - pushing the video capture button. In this moment the secondary form will display each frame separately.
2.1 - once the webcam starts, the optimization/filtration algorithm as well as the algorithm of detection of the laser fascicle are applied to the captured frames from the secondary form.

2.2 - the user may choose an image filtering color;

3.1 - activate the display option of the contextual menu of the mouse;

3.2 - the new image with the laser fascicle will be displayed only when the option ,Activate Laser Detection" is selected. Now the new coordinate of the mouse will be the one that is detected by the algorithm, meaning the coordinates of the laser fascicle that are transposed to the resolution of the current monitor. 


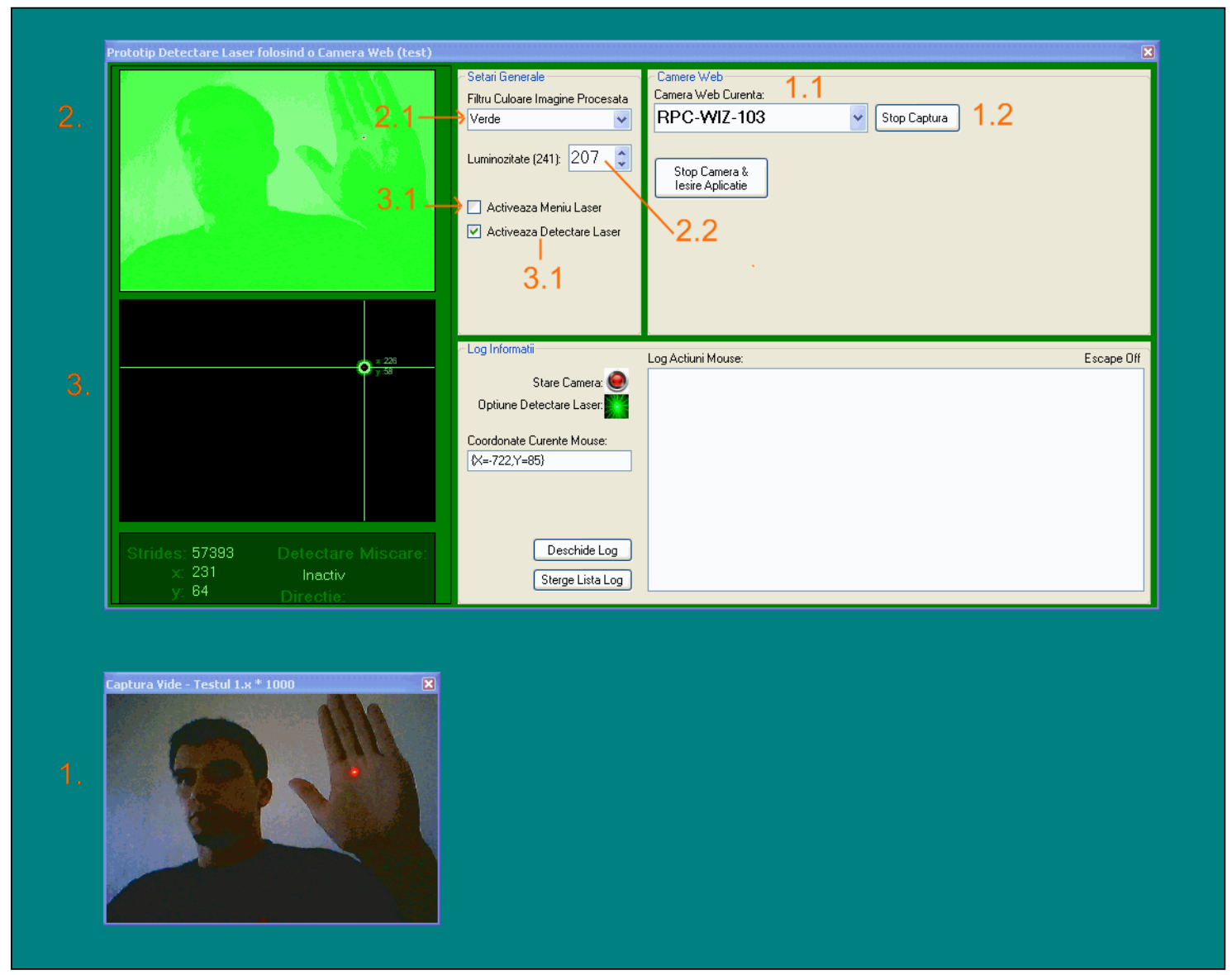

Fig. 10. The application interface

When the application is on, its control will be taken by the light fascicle (meaning the laser pointer). Keeping the laser fascicle only for a few seconds at a certain coordinate will have as an effect the activation of the contextual menu. In the testing phase of this prototype, it was noticed that it is necessary a new intervention on the algorithm in order to offer a more accurate control to the user. This principle was carefully analyzed because it is desired for the user to control the application and not the other way around.

Therefore, if the laser fascicle is pointed towards a certain point, the algorithm will detect the new position and it will graphically display the new point.

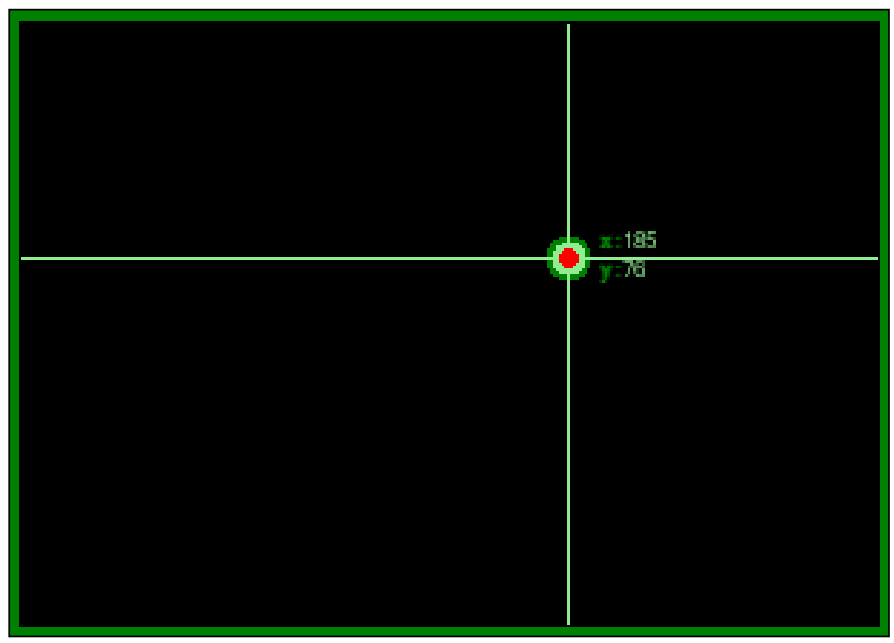

Fig. 11. The display panel of the coordinate of the laser fascicle 
This change of the coordinate will be applied to the mouse according to the width/height of the active monitor (resolution).

\section{Conclusions}

The integration of the information technologies is one of the starting points for the future systems. As we could see in this paper, the hardware and software integration can generate new features for the final users. We intend to develop the present prototype and to implement a better control of the laser fascicle by processing the light color and so we want to diversify the possibilities of the application control.

\section{References}

[1] M. Draganescu, "Perspectivele societatii cunoasterii in Romania", 2010. [Online]. Available: http://www.racai.ro/ dragam/AGIR2.pdf

[2] V. Letokhov, C. Abitbol "Progress in optics", Volume 16, Emil Publishing House, Wolf, North-Holland, 2001, p. 239

[3] R. Aldricht, "Laser Fundamentals", 2012. [Online]. Available: http://www.fas.org/man/dod101/navy/docs/laser/fundamentals.htm\#L ASER $\% 20$ THEORY\%20AND\%20OPERATION

[4] T. Bruno, D.N. Svoronos, "CRC Handbook of Fundamental Spectroscopic Correlation Charts", CRC Press, Colorado, USA, 2005

[5] R. Bowden, "Learning Non-linear Models of Shape and Motion. Enhancing tracking using colors", 2011. [Online]. Available:

http://info.ee.surrey.ac.uk/Personal/R.Bo wden/publications/thesis/download/chapt er4.pdf

[6] P. Sudhir, "Seminar of „Image Processing”, Gyan Vihar School of Engineering \& Technology, 2008

[7] J. A. King, "Digital Photography for Dummies", 5th Edition, Wiley Publishing, Canada, 2005

[8] W3C, "Techniques for Accessibility Evaluation and Repair Tools", Working Draft, accessed august 2010. [Online]. Available: http://www.w3.org/TR/AERT

[9] M. Combs, "Color Matrix Basics - Simple Image Color Adjustment", 2003. [Online]. Available: http://www.codeproject.com/KB/GDIplus/colormatrix.aspx

[10] Microsoft Corporation, "Color Matrix Class", 2012. [Online]. Available: http://msdn.microsoft.com/enus/library/system.drawing.imaging.color matrix.aspx

[11] B. Powell, "How to convert a color image to grayscale", 2011. [Online]. Available:

http://www.bobpowell.net/grayscale.htm

[12] Microsoft Corporation, "Lock Bits Method”, 2012. [Online]. Available: http://msdn.microsoft.com/enus/library/5ey6h79d.aspx

[13] Microsoft Corporation, "Unclock Bits Method", 2011. [Online]. Available:http://msdn.microsoft.com/enus/library/system.drawing.bitmap.unlock bits.aspx

[14] Microsoft Corporation, "Bitmap and Image constructor dependencies", 2012. [Online]. Available: http://support. microsoft.com/kb/814675/en-us

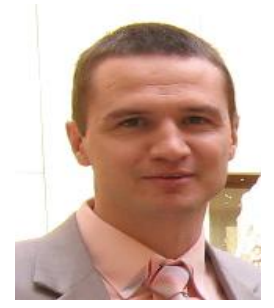

Octavian DOSPINESCU graduated the Faculty of Economics and Business Administration in 2000 and the Faculty of Informatics in 2001. He achieved the $\mathrm{PhD}$ in 2009 and he has published as author or co-author over 30 articles. $\mathrm{He}$ is author and co-author of 10 books and teaches as a lecturer in the Department of Information Systems of the Faculty of Economics and Business Administration, University Alexandru Ioan Cuza, Iasi. Since 2010 he has been a Microsoft Certified Professional, Dynamics Navision, 
Trade\&Inventory Module. He is interested in mobile devices software, computer programming and decision support systems.

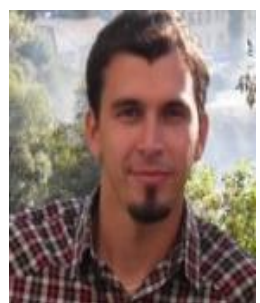

Paul BRODNER graduated the Faculty of Economics and Business Administration, Alexandru Ioan Cuza University of Iasi. He is interested in developing smart systems, mobile applications and he also implemented integrated systems. He is co-author of a C\# Programming Book published in 2009. Now he works for OSF Global Services and implements new technologies for big companies. 